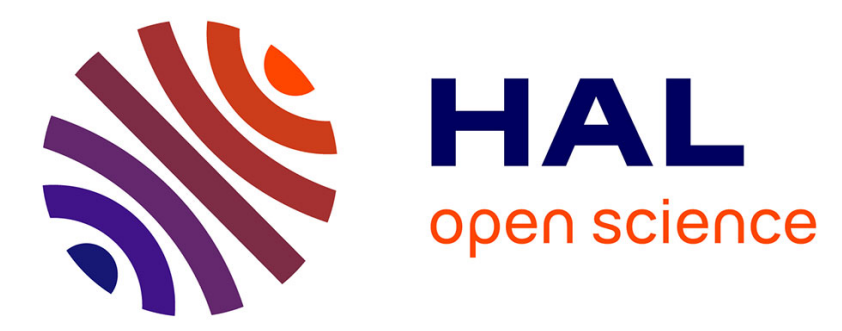

\title{
High Dynamic Engine-Dynamometer Identification and Control
}

\author{
Abderrahim Lamara, Patrick Lanusse, Alain Charlet, Dominique Nelson \\ Gruel, Guillaume Colin, Antoine Lesobre, Alain Oustaloup, Yann Chamaillard
}

\section{> To cite this version:}

Abderrahim Lamara, Patrick Lanusse, Alain Charlet, Dominique Nelson Gruel, Guillaume Colin, et al.. High Dynamic Engine-Dynamometer Identification and Control. 19th IFAC World Congress, Aug 2014, Cape Town, South Africa. pp.5217-5222. hal-01062633

\section{HAL Id: hal-01062633 \\ https://hal.science/hal-01062633}

Submitted on 10 Sep 2014

HAL is a multi-disciplinary open access archive for the deposit and dissemination of scientific research documents, whether they are published or not. The documents may come from teaching and research institutions in France or abroad, or from public or private research centers.
L'archive ouverte pluridisciplinaire HAL, est destinée au dépôt et à la diffusion de documents scientifiques de niveau recherche, publiés ou non, émanant des établissements d'enseignement et de recherche français ou étrangers, des laboratoires publics ou privés. 


\title{
High Dynamic Engine-Dynamometer Identification and Control
}

\author{
A. Lamara ${ }^{*, * * \&^{* * *}, \text { P. Lanusse }}{ }^{* *}$, A. Charlet ${ }^{*}$, D. Nelson Gruel ${ }^{*}$, G. Colin*, A. Lesobre ${ }^{* * *}$, \\ A. Oustaloup **, Y. Chamaillard* \\ * University of Orléans, PRISME, EA4229 \\ 8, rue Léonard de Vinci, 45072 Orléans, France \\ **Bordeaux INP, IMS CNRS UMR5218 \\ University of Bordeaux, IMS CNRS UMR5218 \\ 351 cours de la libération, 33405 Talence, France \\ *** D2T, 11 rue Denis Papin 78190 Trappes, France \\ abderrahim.lamara@d2t.fr
}

\begin{abstract}
In this paper, a high dynamic test-bed control problem for high dynamic vehicle simulation is studied in order to improve the tracking of speed and torque. As the system is nonlinear and square $(2 \times 2)$ with two inputs and two outputs, a multi-input multi-output (MIMO) system-identification problem is discussed. The test-bed data are processed using a fast frequency domain methodology to build a nominal linear plant and a perturbation model. Based on this perturbed plant, the CRONE Control-System Design approach (a fractional order of differentiation based methodology) is used to design a robust MIMO controller, which maintains performance around a wide set of operating points. Finally, experimental results show the relevance of the proposed methodology.
\end{abstract}

Keywords: Identification, multivariable control, CRONE, nonlinear systems, robust control.

\section{INTRODUCTION}

The need to reduce pollutant emissions and improve vehicle performances involves the development of new strategies for engine control. At present, the engine performance is checked and validated step by step throughout the whole development process of the engine and its integration in a completed vehicle. Subsequently, the engine is calibrated on-board the vehicle. However, a failure in one step of the development process means a new test process. Today, the automotive industry needs a simpler and shorter process to reduce the development time and hence the cost. To deal with this challenge, a high dynamic test-bed is required, in order to reproduce the road behavior and the gearbox ratio change. The control system should ensure good tracking of speed and torque.

Several aspects of test bed control have tackled in previous studies. In Chauvin et al. (2009), the system is firstly modeled by physical equations and then an observer is developed for dynamic estimation of the torque produced by the engine. Priesner et al. (2012) presented a model predictive disturbance compensation control concept for an industrial combustion engine test-bed. As the torque control of the vibrating shaft requires a high bandwidth, a short sampling time was used. Based on an approximate model of the test-bed, modified in order to cope with non-modeled dynamics and nonlinearities, Passenbrunner et al. (2011) designed a dynamic control law for the speed and torque control of an internal combustion engine test-bed. Discretetime control design was studied in Shona Laila et al. (2007), where a model reference approach was used for the systemcontrol design, to make the controller less sensitive to sampling and to keep sampled-data tracking performance as close as possible to the continuous-time tracking performance. Gruenbacher and Del Re (2008) presented a robust inverse control for a combustion engine test-bed. Gruenbacher and Marconi (2009) developed an adaptive internal model in order to control a combustion engine testbed in idle mode. In this case the combustion engine is declutched from the power train and hence its only load is the drag (friction) torque. An inverse control torque of hydrodynamic dynamometers for a combustion engine testbed was presented in Passenbrunner et al. (2011), where the dynamometer is modeled by a nonlinear static map combined with linear dynamics, and the model obtained gives the relation between the valve position and the dynamometer torque, using the nonlinear static map as a feed-forward to define the valve position based on the torque demand. Then, a feedback controller is used to compensate uncertainties and disturbance effects. Bunker et al. (1997) developed a robust multivariable controller to control a highly coupled Diesel engine-dynamometer system. As the load has a faster dynamic behavior than the combustion engine, the high dynamics of the speed control loop is naturally filtered by the torque control loop. However, the speed is highly affected by the torque variation. A large change in load command would cause engine stall.

This paper proposes a faster methodology for test-bed identification and control. For a set of operating points, the dynamics of the system are approximated to a set of linear plants using a fast frequency-domain method. The defined perturbed frequency-domain plant is then used to design a multivariable robust controller.

The test-bed system is described in section 2, and the frequency-domain methodology is presented and then used to approximate the system dynamics to a linear perturbed plant. Section 3 explains how the CRONE Control-System Design (CSD) methodology uses the perturbed plant to design a 
robust multivariable controller. In the last section, results from experiments are compared and discussed.

\section{SYSTEM IDENTIFICATION}

\subsection{System description}

The test-bed used in this paper consists of a simulated engine to be tested and a load, which can be provided by a brake or a dynamometer. It uses a classic configuration (fig. 1) with two real asynchronous machines. The first one simulates a dynamometer. The second machine is associated to a nonlinear model with a variable time constant and variable time-delay, which simulates the behavior of a combustion engine.

The power of the asynchronous machines is about $11 \mathrm{~kW}$, with a max speed of $1500 \mathrm{rpm}$. The two machines are connected via a transmission and power is supplied by the ACS800 ABB drive. The whole system is piloted by the MORPHEE simulator. MORPHEE is a D2T automation system able to execute automatic cycles, save data during tests and integrate Simulink and AMEsim models to be executed in real time.

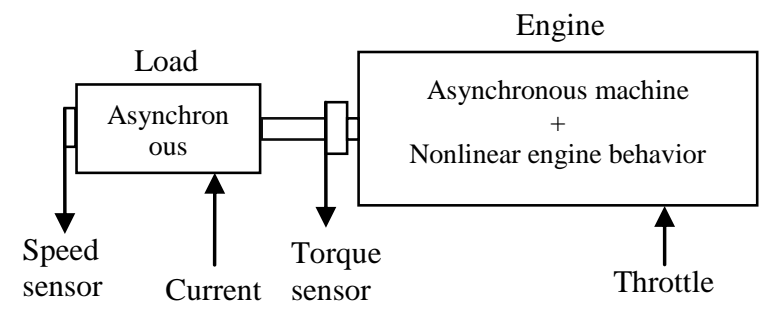

Fig. 1: Test-bed configuration

Dynamic combustion engine test-beds are used for many purposes, such as calibration, driving cycles, control development and research. In industry, engineers need to change the engine and the test-bed configuration several times, and then new controllers are required to deal with new challenges. The classical solution is PID tuning around some operating points, but robustness is not guaranteed and such a procedure is very time consuming. In Blumenschein et al. (2013) an adaptive model of the test bench for heavy duty internal combustion engines was presented in order to ease observer design and to compensate for the lack of torque measurement.

Once the engine has been changed, a new test-bed control system is required. The speed can be controlled by the load (dynamometer or brake) and the torque by the engine, the load is highly coupled with the engine through the shaft and any change in the load behavior is observed as a perturbation on the engine side and vice versa. Thus, a MIMO CSD approach seems to be necessary. In the present study, a model of the test-bed was developed based on physical equations and maps. This representative model was used for the development and validation of our proposed methodology. As the load is an electrical machine, its model is approximated to a linear model. The ABB drive is modeled by a simple map combined with a first order filter with timedelay. Nonlinear modeling of the internal combustion engine is unavoidable because of inner nonlinear control loops and various nonlinear mechanical systems (crankshaft, cylinder etc). In this paper, the engine is simply modeled using the same linear model of the load, and an operating point dependent first order filter with a time-constant varying from $0.15 \mathrm{~s}$ to $1 \mathrm{~s}$ and a time-delay varying from $0 \mathrm{~s}$ to $0.2 \mathrm{~s}$.

\subsection{System identification}

The frequency-domain CSD approach used in this work requires high fidelity linear dynamic models that approximate the underlying dynamics of the engine. Thus, a frequency domain system identification methodology is used to obtain a linear time-invariant model that approximates the dynamics of the test-bed. A multisine signal is used for input excitation. The use of this broadband signal allows faster frequency domain identification. Based on the multisine frequency response, its spectrum is within the defined band which gives a good approximation of the system dynamics (see Lamara et al. 2012). The multisine signal is a linear combination of sinusoids which is given by:

$$
u(t)=\sum_{k=1}^{N} A_{k} \cos \left(2 \pi f_{k} t+\varphi_{k}\right)
$$

where:

$$
\begin{aligned}
& N: \text { is the signal harmonics number } \\
& f_{k}: \text { is the frequency of harmonic } k \\
& A_{k}: \text { is the amplitude of harmonic } k \\
& \varphi_{k}: \text { is the initial phase of harmonic } k
\end{aligned}
$$

The amplitude $A_{k}$ is chosen in such way that the system gives a linear response. In this study the torque variation must be no more than $5 \%$ of the maximum torque at the corresponding speed in order to avoid breaking the shaft. The frequency $f_{k}$ is chosen such that the time-invariant nominal model approximates the most important linear dynamics of the real system. For a good controller design, the frequency $f_{N}$ must be greater than the desired cut-off frequency or resonant frequency, of the open loop $\beta_{0}$. The excitation signal is chosen with a sampling frequency of $1 \mathrm{kHz}$ and a frequency range from $0.1 \mathrm{~Hz}$ to $20 \mathrm{~Hz}$. An automatic cycle is developed, using MORPHEE, to execute the identification process around twenty well-chosen (and arbitrary) operating points.

The test-bed studied is a MIMO system with two inputs and two outputs $(2 \times 2)$.

$$
\left[\begin{array}{l}
Y_{1} \\
Y_{2}
\end{array}\right]=\left[\begin{array}{ll}
G_{11} & G_{12} \\
G_{21} & G_{22}
\end{array}\right]\left[\begin{array}{l}
U_{1} \\
U_{2}
\end{array}\right]
$$

The identification of each element of the transfer matrix consists in exciting only one input, while the second input keeps its mean value. Each element of the frequencyresponse transfer matrix is calculated using the Fast Fourier Transformation (FFT) of the ratio of the cross-correlation between output and input $\tau_{y x}$ divided by the autocorrelation of input $\tau_{x x}$ as in Lamara et al. (2012). Each input-output transfer is then described:

$G_{11}:$ from current input $(\%)$ to speed sensor (rpm)
$G_{12}:$ from throttle (\%) to speed sensor (rpm)
$G_{21}:$ from current input $(\%)$ to torque sensor (N.m) 
$G_{22}:$ from throttle (\%) to torque sensor (N.m)
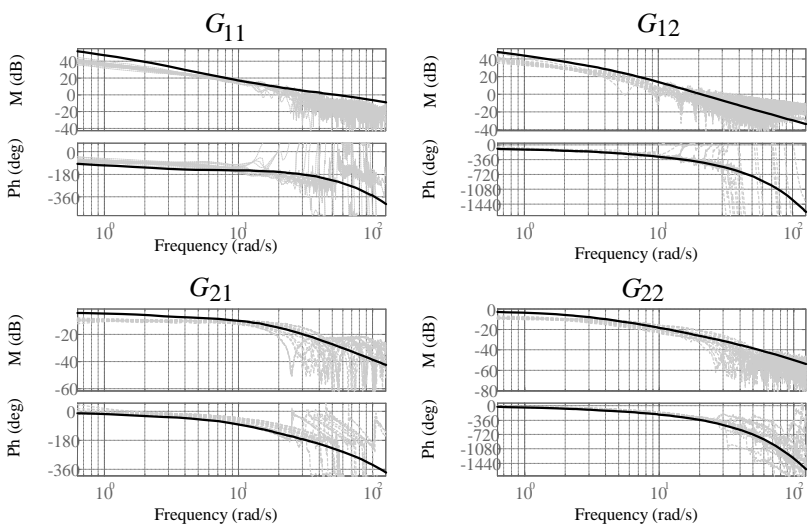

Fig. 2: Bode diagram of the real system around twenty operating points and the defined nominal plant (dark)

The frequency domain identification provides a nonparametric model. For each element of the transfer matrix, the parameters of a fourth-order transfer function with timedelay were used to obtain a nominal parametric model:

$$
G_{i j}=\frac{A\left(s+a_{0}\right)\left(s+a_{1}\right)}{\left(s+b_{0}\right)\left(s+b_{1}\right)\left(s+b_{2}\right)\left(s+b_{3}\right)} e^{-\tau s}
$$

Each element of the nominal transfer matrix is selected using a minimum phase criterion (dark plot on fig. 2). In the next step, the nominal plant and the whole set of calculated frequency-responses are used to design a robust multivariable controller, which maintains performances around a wide set of operating points.

\section{CONTROL METHODOLOGY}

The objective of the CRONE control system design is to robustify the closed loop dynamic performance through either a robust damping factor or a robust resonant peak control (Oustaloup et al. 1995) based on the unity-feedback configuration (fig. 3).

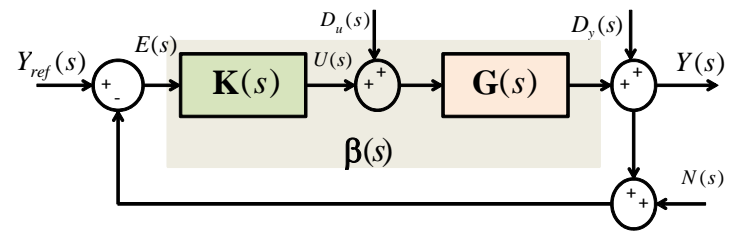

Fig. 3: Unity-feedback configuration for CRONE approach

$D_{\mathrm{u}}(s)$ and $D_{\mathrm{y}}(s)$ are disturbances on input and output. $N(s)$ is measurement noise. $E(s)$ is the error and $U(s)$ is the control effort.

Fractional differentiation is used to define the nominal and diagonal open-loop transfer function of square systems $(n \times n)$ :

$$
\boldsymbol{\beta}_{0}(s)=\mathbf{G}_{0}(s) \mathbf{K}(s)=\left[\begin{array}{ccc}
\beta_{011}(s) & \cdots & 0 \\
\vdots & \ddots & \vdots \\
0 & \cdots & \beta_{0 n n}(s)
\end{array}\right]
$$

Once $\boldsymbol{\beta}_{0}(s)$ has been optimized, the controller can be obtained from

$$
\boldsymbol{K}(s)=\mathbf{G}_{0}^{-1}(s) \boldsymbol{\beta}_{0}(s)
$$

where $\mathbf{G}_{0}^{-1}(s)$ is the inverse of the nominal plant $\mathbf{G}_{0}(s)$.

$$
\text { Let } \begin{aligned}
\mathbf{G}_{0}(s)= & {\left[\begin{array}{ccc}
g_{11}(s) & \cdots & g_{1 n}(s) \\
\vdots & \ddots & \vdots \\
g_{n 1}(s) & \cdots & g_{n n}(s)
\end{array}\right] } \\
& \text { With } g_{i j}(s)=h_{i j}(s) e^{-L_{i j} s}
\end{aligned}
$$

- $\quad h_{i j}(s)$ : is a strictly proper time-delay free transfer function,

$$
\text { - } \quad L_{i j} \in \mathbb{R}^{*+} \text {. }
$$

Nelson et al. (2008) defined $\mathbf{P}(s)$ :

$\mathbf{G}_{0}^{-1}(s)=\mathbf{P}(s)=\left[\begin{array}{ccc}P_{11}(s) e^{\gamma_{11} s} & \cdots & P_{1 n}(s) e^{\gamma_{1 n} s} \\ \vdots & \ddots & \vdots \\ P_{n 1}(s) e^{\gamma_{n 1} s} & \cdots & P_{n n}(s) e^{\gamma_{n n} s}\end{array}\right]$

where $\mathbf{P}(s)$ is the non-zero transfer function and $\gamma_{i j} \in \mathbb{R}^{*+}$.

Each element of the diagonal open-loop matrix $\boldsymbol{\beta}_{0}(s)$ is based on the third generation CRONE single-input singleoutput (SISO) methodology. The principle of this methodology is to optimize the parameters of the nominal SISO open-loop transfer function $\beta_{0}(s)$ that includes bandlimited complex fractional order integration over a frequency range $\left[\omega_{A}, \omega_{B}\right]$. The complex fractional order, $a+\mathrm{i} b$, enables a straight line of any direction to be created in the Nichols chart which is called the generalized template (fig. 4).

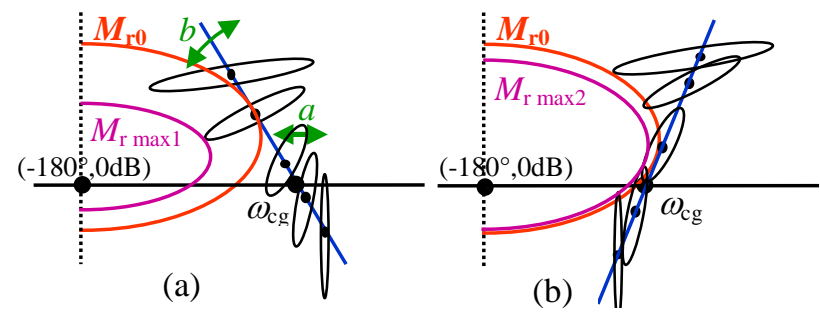

Fig. 4: (a) any generalized template, (b) optimal template

In the CRONE SISO methodology, the nominal open-loop transfer function is defined as a multiplication of three transfer functions given by:

where

$$
\beta_{0}(s)=\beta_{\mathrm{l}}(s) \beta_{\mathrm{m}}(s) \beta_{\mathrm{h}}(s)
$$

- $\quad \beta_{1}(s)$ is an integer order $n_{1}$ proportional integrator

$\beta_{\mathrm{l}}(s)=C_{\mathrm{l}}\left(\frac{\omega_{-N^{-}}}{s}+1\right)^{n_{\mathrm{l}}}$

Order $n_{1}$ has to be set to manage the accuracy provided by the control system.

- $\quad \beta_{\mathrm{m}}(s)$ is a set of band-limited generalized templates

$\beta_{\mathrm{m}}(s)=\prod_{k=-N^{-}}^{N^{+}} \beta_{\mathrm{m} k}(s)$

with:

$\beta_{\mathrm{m} k}(s)$

$$
\begin{gathered}
=C_{k}^{\operatorname{sign}\left(b_{k}\right)}\left(\alpha_{k} \frac{1+\frac{s}{\omega_{k+1}}}{1+\frac{s}{\omega_{k}}}\right)^{a_{k}}\left(\Re e_{/ i}\left\{\left(\alpha_{k} \frac{1+\frac{s}{\omega_{k+1}}}{1+\frac{s}{\omega_{k}}}\right)^{i b_{k}}\right\}\right)^{-q_{k} \operatorname{sign}\left(b_{k}\right)} \\
\alpha_{k}=\left(\frac{\omega_{k+1}}{\omega_{k}}\right)^{1 / 2} \text { for } k \neq 0 \text { and } \alpha_{0}=\left(\frac{1+\left(\frac{\omega_{\mathrm{r}}}{\omega_{0}}\right)^{2}}{1+\left(\frac{\omega_{\mathrm{r}}}{\omega_{1}}\right)^{2}}\right)^{\frac{1}{2}}
\end{gathered}
$$


$N^{-}$and $N^{+}$can be different from 0 to increase the number of tuning parameters used for the open-loop shaping.

- $\quad \beta_{\mathrm{h}}(s)$ is the low-pass filter of integer $n_{\mathrm{h}}$

$\beta_{\mathrm{h}}(s)=C_{\mathrm{h}}\left(\frac{s}{\omega_{N^{+}}}+1\right)^{n_{\mathrm{h}}}$

Order $n_{\mathrm{h}}$ has to be set to obtain a proper or bi-proper control system.

$\omega_{\mathrm{k}}$ are corner frequencies and the gains $C_{k}, C_{\mathrm{l}}$ and $C_{\mathrm{h}}$ are such that $\omega_{\mathrm{r}}$ is the nominal closed-loop resonant frequency.

To make the controller achievable and the closed loop fully stable for unstable systems, non-minimum phase SISO systems or systems with time-delay, the open-loop transfer function must include the nominal time-delay and right half plane zeros and poles of $G_{0}(s)$ (Lanusse and Oustaloup 2005).

Frequency uncertainty domains were defined from the multiplicative uncertainty of the SISO open-loop frequency response in the Nichols chart. This multiplicative uncertainty is invariant and equal to the plant uncertainty:

$$
\beta(s)=G(s) K(s)=G_{0}(s) \Delta_{\mathrm{m}} K(s)=\beta_{0}(s) \Delta_{\mathrm{m}}
$$

In Fig. 4 both generalized templates (a and b) tangent the same required nominal resonant peak $M_{\mathrm{r} 0}$. Fig. 4 shows that the resonant peak $M_{\mathrm{r}}$ variation of the complementary sensitivity function is reduced by optimizing the position of the generalized template in the Nichols chart (fig. 4.a and 4.b). The robustness cost function is given by

$$
J=\left(M_{\mathrm{r}_{0}}-\inf \left|M_{\mathrm{r}}\right|\right)^{2}+\left(\sup \left|M_{\mathrm{r}}\right|-M_{\mathrm{r}_{0}}\right)^{2}
$$

where $M_{\mathrm{r}_{0}}$ is the nominal value of the resonant peak. Its minimization is accomplished while respecting closed loop frequency-domain constraints for all plants $G$ and for $\omega \in$ $\mathbb{R}^{+}$.

For $n \times n$ MIMO systems, the aim of this methodology is to find a diagonal open-loop transfer matrix with $n$ fractional order transfer elements. This transfer matrix is parameterized so as to satisfy the following objectives:

- perfect decoupling for the nominal plant

- required nominal stability margins of the closed loops

- accuracy specification at low frequency

- specifications on the $n$ control efforts at high frequencies.

The resonant peaks taken into account in a cost function based on (11) are those of the diagonal elements of the perturbed complementary sensitivity transfer function matrix T. The controller elements are obtained from relations (5) and (7):

$$
K_{i j}(\mathrm{j} \omega)=P_{i j}(\mathrm{j} \omega) e^{\gamma_{i j} \mathrm{j} \omega} \beta_{0 j j}(\mathrm{j} \omega)
$$

As $\beta_{j j}(s)$ are fractional order transfer functions, the rational transfer functions $K_{\mathrm{Rij}}(s)$ are obtained by identifying the ideal frequency responses $K_{i j}(\mathrm{j} \omega)$ by low-order transfer functions:

$$
K_{\mathrm{R}_{i j}}(s)=\frac{B(s)}{A(s)}
$$

where $B(s)$ and $A(s)$ are polynomials of specified integer degrees $n_{\mathrm{B}}$ and $n_{\mathrm{A}}$. All the frequency-domain systemidentification techniques can be used (for instance Oustaloup et al. 2000 or Oustaloup et al. 2002). Whatever the complexity of the control problem, it is easy to find satisfactory values of $n_{\mathrm{B}}$ and $n_{\mathrm{A}}$ without a decrease in performances.

Since $\mathbf{G}_{0}(s)$ is a full matrix transfer function, from (5) the controller will be a full MIMO controller (fig. 5). All the elements of the transfer matrix will be taken into account for open-loop optimization. The decoupling and stabilizing controller $K$ exists if and only if the following hypotheses are verified:

$$
\begin{aligned}
& H_{1}:\left[\mathbf{G}_{0}(s)\right]^{-1} \text { exists, }(14) \\
& H_{2}: Z_{+}\left[\mathbf{G}_{0}(s)\right] \cap P_{+}\left[\mathbf{G}_{0}(s)\right]=0
\end{aligned}
$$

where $Z_{+}\left[\mathbf{G}_{0}(s)\right]$ and $P_{+}\left[\mathbf{G}_{0}(s)\right]$ indicate the positive real part zero and pole sets.

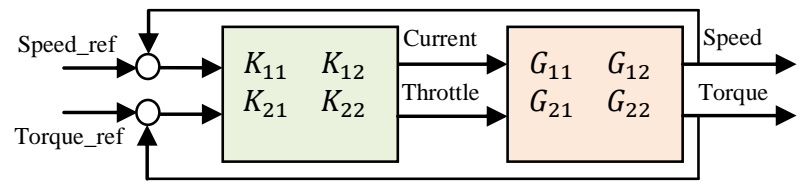

Fig. 5: Full MIMO control for the $2 \times 2$ test bed square system

Even if the closed-loop perturbed transfer matrices $\mathbf{T}(s)$ and $\mathbf{S}(s)$ are not diagonal, each diagonal element $T_{i i}(s)$ and $S_{i i}(s)$ can be interpreted as a closed-loop transfer function resulting from a SISO open-loop transfer function $\beta_{i i}(s)$ called equivalent open-loop transfer function:

$$
\beta_{i i}(s)=\frac{T_{i i}(s)}{1-T_{i i}(s)}=\frac{1-S_{i i}(s)}{S_{i i}(s)}
$$

Taking into account this equivalent perturbed open-loop transfer function, the optimization of all diagonal elements $\beta_{0_{i i}}(s)$ is achieved by minimizing the robustness cost function:

where

$$
J=\sum_{i=1}^{n}\left(M_{\mathrm{r}_{\max _{i}}}-M_{\mathrm{r}_{\min _{i}}}\right)^{2}
$$

$$
\begin{aligned}
-M_{\mathrm{r}_{\max _{i}}} & =\max _{\mathbf{G}} \sup \left(T_{i i}(\mathrm{j} \omega)\right) \\
-M_{\mathrm{r}_{\min _{i}}} & =\min _{\mathbf{G}} \sup \left(T_{i i}(\mathrm{j} \omega)\right)
\end{aligned}
$$

where $\mathbf{G}$ is the nominal or perturbed plant, and while respecting the frequency-domain inequalities below for $\omega \in \mathbb{R}$ and $i, j \in N$ :

$$
\begin{aligned}
& \sup _{G}\left|T_{i j}(\mathrm{j} \omega)\right| \leq T_{i j_{\mathrm{u}}}(\omega) \\
& \inf _{G} \mid T_{i j}(\mathrm{j} \omega) \geq T_{i j_{\mathrm{l}}}(\omega) \\
& \sup _{G}\left|S_{i j}(\mathrm{j} \omega)\right| \leq S_{i j_{\mathrm{u}}}(\omega) \\
& \sup _{G} \mid K S_{i j}(\mathrm{j} \omega) \leq K S_{i j_{\mathrm{u}}}(\omega) \\
& \sup _{G} \mid S G_{i j}(\mathrm{j} \omega) \leq S G_{i j_{\mathrm{u}}}(\omega)
\end{aligned}
$$

with

$$
\begin{aligned}
& \mathbf{S}(s)=[I+\mathbf{G}(s) \mathbf{K}(s)]^{-1}=\left[S_{i j}(s)\right]_{1 \leq i, j \leq n} \\
& \mathbf{T}(s)=\mathbf{S}(s) \mathbf{G}(s) \mathbf{K}(s)=\left[T_{i j}(s)\right]_{1 \leq i, j \leq n}
\end{aligned}
$$


The uncertainties in $\mathbf{G}$ are taken into account by the least conservative method. For this reason, a nonlinear optimization method must be used to find the optimal values of the high-level descriptive parameters of the fractional open-loop and consequently to find an optimal placement of the equivalent open-loop frequency response $\beta_{i i}(j \omega)$.

As for unstable SISO systems, non-minimum phase SISO systems and SISO systems with time-delay, the MIMO openloop transfer function must include some time-delays and right half plane zeros and poles of $\mathbf{G}_{0}(s)$ and $\mathbf{P}(s)$ (Nelson Gruel et al. 2008).

\section{EXPERIMENTAL RESULTS}

The association of the engine with the dynamometer makes the system highly coupled. It is possible to design two controllers to control the speed and the torque separately. In the proposed methodology, the CRONE full-MIMO controller controls the speed and the torque simultaneously.

In order to evaluate the controller performances, a testperformance cycle was developed in MORPHEE (fig. 8 and 9). The user indicates the maximum and the idle speeds, and then set-points are generated automatically by MORPHEE.

Two controllers were tested on the real system, and the controller performances were compared using the testperformance cycle developed. The first controller consists of two PIDs tuned by trial and error using the experimental system. As is customary in industry, the torque control loop is open and the speed PID controller is tuned. Once the speed PID has been tuned, the torque loop is then closed and the torque PID controller is tuned.

The second controller is a CRONE MIMO controller. In the MIMO approach all the elements of the nominal transfer matrix are used for the open-loop optimization. Fig. 6 shows the $\beta_{0_{11}}$ open-loop optimization. A bandwidth of $8 \mathrm{rad} / \mathrm{s}$ was chosen to guarantee fast tracking of the speed set-point. As the perturbed frequency responses are noisy, the resonant peak $T_{0_{11}}$ can be considered to be about $2 \mathrm{~dB}$ (the frequency response inside the low stability area is considered as noise) which makes the controller robust around the identified operating points.

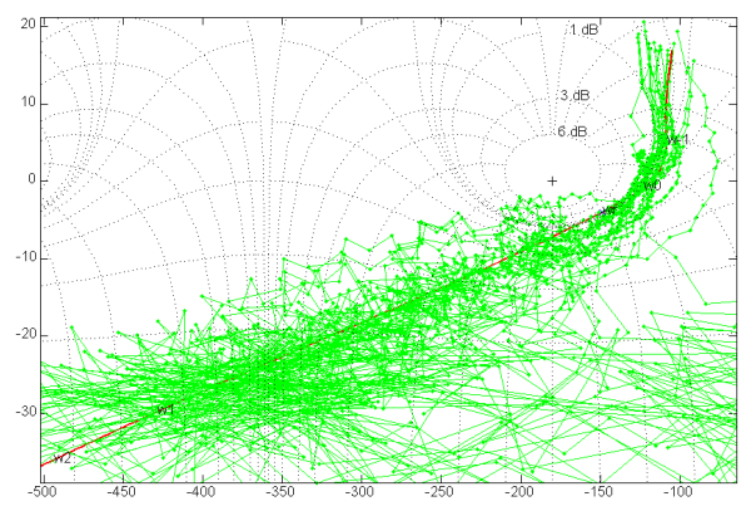

Fig. 6: $\boldsymbol{\beta}_{\mathbf{0}_{11}}$ open-loop optimization and equivalent openloop $\boldsymbol{\beta}_{11}$
Fig 7 shows the $\beta_{0_{22}}$ open-loop optimization. A bandwidth of $1.4 \mathrm{rad} / \mathrm{s}$ was chosen. The resonant peak $T_{0_{22}}$ for this openloop is about $1 \mathrm{~dB}$. The two open-loops $\beta_{0_{11}}$ and $\beta_{0_{22}}$ are optimized simultaneously. Any modification in one of the nominal loops can be seen on the perturbed second loop, which takes interaction phenomena into account.

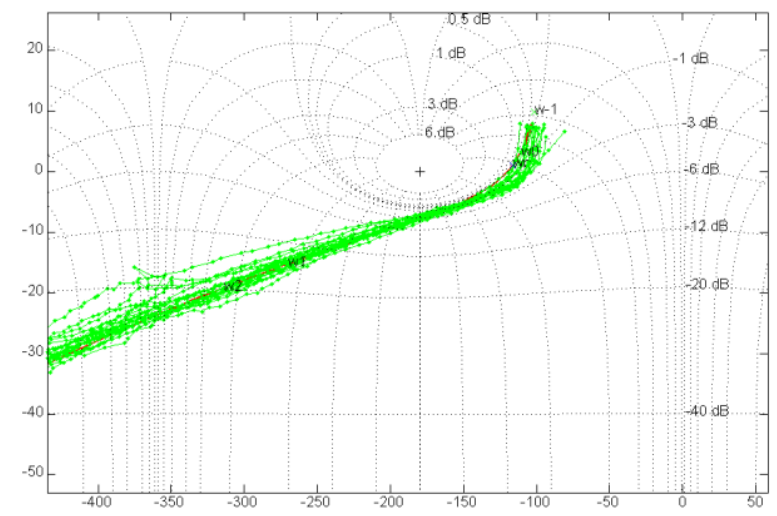

Fig. 7: $\boldsymbol{\beta}_{\mathbf{0}_{22}}$ open-loop optimization and equivalent openloop $\boldsymbol{\beta}_{\mathbf{2 2}}$

For the test-performance cycle, the speed step-response from $440 \mathrm{rpm}$ to $800 \mathrm{rpm}$ and then from $800 \mathrm{rpm}$ to $1160 \mathrm{rpm}$ is shown in fig. 8. From the comparison between the PID and the CRONE controllers, speed set-points tracking, it can be seen that the CRONE overshoot is less than the PID overshoot, but that the settling times are very close, despite the fact that the PID rise time is less than the CRONE rise time.
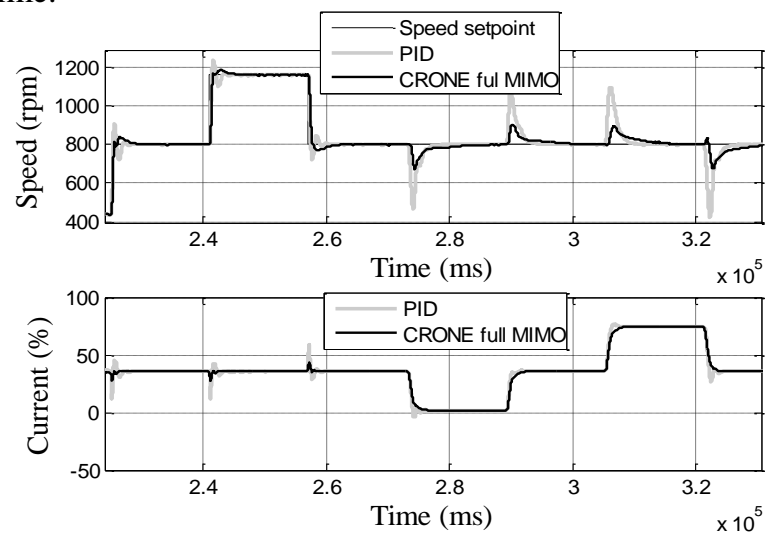

Fig. 8: Speed set-points tracking and current control effort
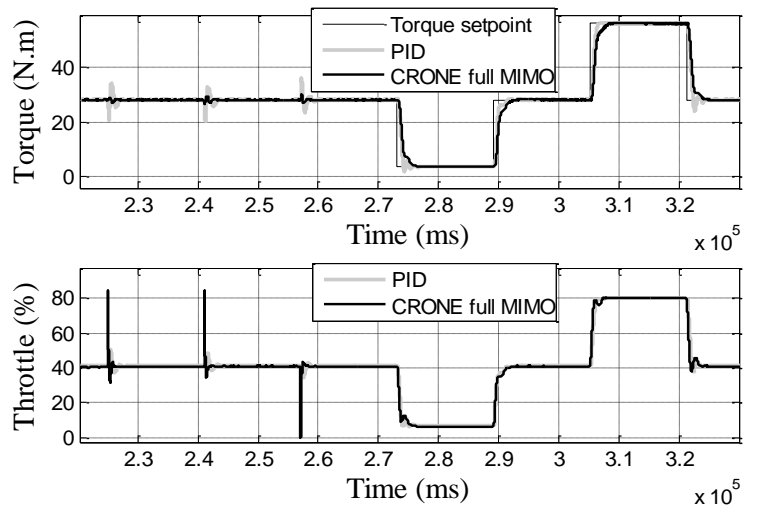

Fig. 9: Torque set-point tracking and throttle control effort 
Concerning the control efforts, the CRONE full MIMO effort is less than that of the PID controller. After the speed step, the CRONE full MIMO tries to reach the setpoint by acting simultaneously on the current and the throttle, whereas the PID uses only the current effort to reach the speed setpoint. Fig. 9 shows the torque step-response from $28 \mathrm{Nm}$ to $3.5 \mathrm{Nm}$, from $3.5 \mathrm{Nm}$ to $28 \mathrm{Nm}$ and from $28 \mathrm{Nm}$ to $56 \mathrm{Nm}$. It reveals that for this operating point, the torque responses are extremely close. For both outputs, the CRONE MIMO controller ensures the best rejection of coupling phenomena.

The torque response shown in fig. 10 presents a robustness problem observed for a torque step from $56 \mathrm{Nm}$ to $28 \mathrm{Nm}$, but now for a speed of $440 \mathrm{rpm}$. The instability of the PID responses can be attributed to the fact that the operating point chosen for the PID tuning was very different from the one tested here.
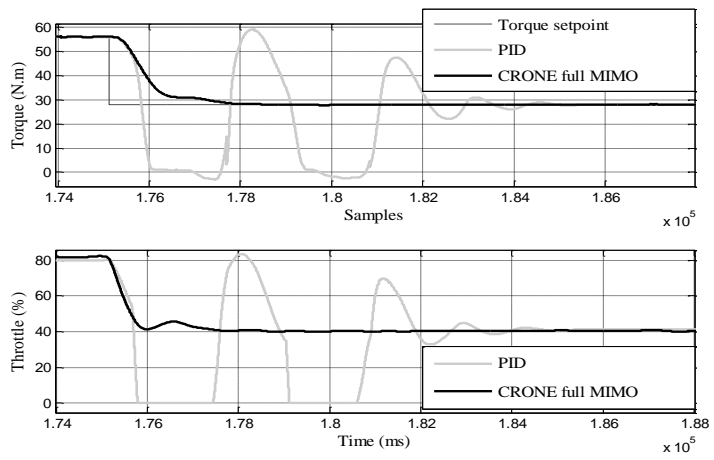

Fig. 10: Robustness problem during a torque step

For the CRONE full MIMO controller, robustness is guaranteed around all the operating points.

\section{CONCLUSION}

A full methodology is presented in this paper for multivariable system-identification and robust controller design. The identification problem is discussed for the proposed nonlinear engine-dynamometer system. After data processing, a nominal plant is defined in order to approximate the most important dynamics of the highly coupled time-varying system, all the calculated frequency responses being taken into account as the plant perturbation model. The CRONE MIMO methodology demonstrates how the robust controller is designed using the defined perturbed plant. The CRONE full MIMO controller designed ensures good set-points tracking and good robustness. The results show the relevance of the proposed methodology. This methodology will be used to design a controller for a high dynamic engine-dynamometer system with a Diesel engine.

\section{REFERENCES}

Blumenschein, J., Schrangl, P., Passenbrunner, E. Trogmann, H., and del Re, L. (2013). Easily Adaptable Model of Test Benches for Internal Combustion Engines. European Control Conference (ECC). Zürich, Switzerland.

Bunker, B.M., Franchek, M.A., member IEEE., and Thomason, B.E. (1997). Robust Multivariable Control of an Engine-Dynamometer System. IEEE Transactions on control systems technology, vol. 5, no. 2.

Chauvin, J., Chasse, A. (2009). Dynamic periodic observer for a combustion engine test bench, Joint 48th IEEE Conference on Decision and Control and 28th Chinese Control Conference. Shanghai, P.R. China.

Gruenbacher, E., Del Re, L. (2008). Robust Inverse Control for Combustion Engine Test Benches. American Control Conference Westin Seattle Hotel, Washington, USA.

Gruenbacher, E., Marconi, L. (2009). Idle Mode Control on a Combustion Engine Test Bench via Internal Mode Control. American Control Conference. Hyatt Regency Riverfront, St. Louis, MO, USA.

Lamara, A., Colin, G., Lanusse, P., Chamaillard, Y. (2012). Decentralized robust control-system for a non-square system, the air-path of a turbocharger Diesel engine. IFAC workshop on engine and powertrain control, simulation and modeling. Rueil-Malmaison, France.

Lanusse, P., and Oustaloup, A. (2005). Control Of TimeDelay Systems Using Robust Fractional-Order Control And Robust Smith Predictor Based Control. ASME International Design Engineering Technical Conferences and Computers and Information in Engineering Conference. Long Beach, California.

Nelson-Gruel, D., Lanusse, P., Oustaloup, A. (2008). Decentralized CRONE control of mxn multivariable system with time-delay. Fractional Differentiation and its Applications. Ankara, Turkey.

Oustaloup, A., Mathieu, B., Lanusse, P., (1995). CRONE control of resonant plants: application to a flexible transmission. European Journal of Control, Vol. 1, $\mathrm{n}^{\circ} 2$, pp. 113-121.

Oustaloup, A., Melchior, P., Lanusse, P., Cois, O., and Dancla, F. (2000). The CRONE toolbox for Matlab. IEEE International Symposium on Computer-Aided Control System Design. Anchorage, Alaska.

Oustaloup, A., Lanusse, P., Levron, F. (2002). Frequency synthesis of filter using the functions of Viète's roots. IEEE Transactions on Automatic Control, Vol. 47, n ${ }^{\circ}$, pp. 837-841.

Passenbrunner, T.E., Sassano, M., and Del Re, L. (2011). Approximate Optimal Control of Internal combustion Engine Test Benches. 50th IEEE Conference on Decision and Control and European Control Conference (CDC$E C C)$. Orlando, FM, USA.

Passenbrunner, T.E., Sassano, M., Trogmann, H., del Re, L., Paulweber, N., Schmidt, M, and Kokal, H. (2011). Inverse torque control of hydrodynamic dynamometers for combustion engine test benches. American Control Conference. San Francisco, CA, USA.

Priesner, R., Westermayer, C., Jakubek, S., and Bauer R. (2012). Predictive Control With Active Disturbance Rejection for Elastic Drive Systems. 7th IEEE Conference of industrial Electronics and Applications (ICIEA).

Shona Laila, D., Grünbacher, A. (2007). Discrete-time control design for setpoint tracking of a combustion engine test bench. Proceedings of the 46th IEEE Conference on Decision and Control. New Orleans, LA, USA. 